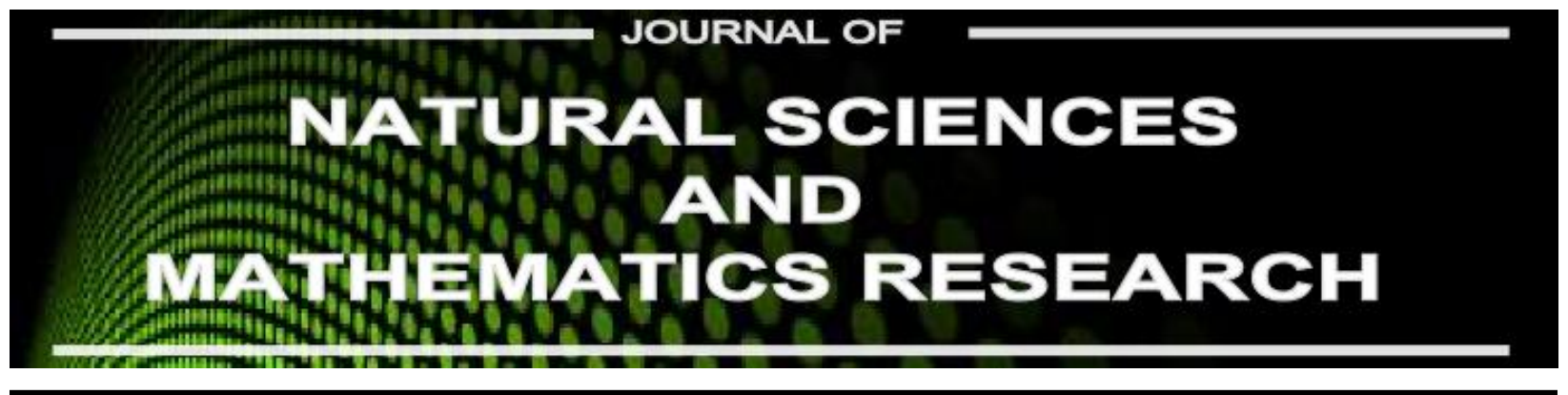

Available online at http://journal.walisongo.ac.id/index.php/jnsmr

\title{
The Solution Of Nonhomogen Abstract Cauchy Problem by Semigroup Theory of Linear Operator
}

\section{Susilo Hariyanto}

Mathematics Department, Faculty of Mathematics and Natural Science, Universitas Diponegoro Semarang, Central Java,Indonesia

Corresponding author: sus2_hariyanto@yahoo.co. id

Recived: 06 November 2016, Revised : 02 December 2016 Accepted: 27 December 2016.

\begin{abstract}
In this article we will investigate how to solve nonhomogen degenerate Cauchy problem via theory of semigroup of linear operator. The problem is formulated in Hilbert space which can be written as direct sum of subset $\operatorname{Ker} M$ and Ran $M^{*}$. By certain assumptions the problem can be reduced to nondegenerate Cauchy problem. And then by composition between invers of operator $M$ and the nondegenerate problem we can transform it to canonic problem, which is easier to solve than the original problem. By taking assumption that the operator $A$ is infinitesimal generator of semigroup, the canonic problem has a unique solution. This allow to define special operator which map the solution of canonic problem to original problem. (C2016 JNSMR UIN Walisongo. All rights reserved.
\end{abstract}

Key words: Degenerate; Nondegenerate; Cauchy Problem; Infinitesimal.

\section{Introduction}

Let us consider the homogen abstract Cauchy problem:

$$
\frac{d}{d t} M z(t)=A z(t), \quad z(0)=z_{0}
$$

For finite dimensional, the problem (1) homogen abstract degenerate Cauchy problem is discussed completely in the book [1], where we can tranform matrices $\mathrm{M}$ and $\mathrm{A}$ to $\mathrm{a}$ common normal form. We also can find many examples, applications to control theory, and references to the earlier literature in his book.

In the infinite dimensional case, it is mentioned in [2] that they treat the singular and degenerate Cauchy problem. In [3-8], the investigates degenerate Cauchy problem in Hilbert space. In his articles, the problem is treated also under the assumption that the Hilbert space of the system can be written as direct sum of the kernel of M (Ker M) and the range of adjoint $\mathrm{M}\left(\operatorname{Ran} \mathrm{M}^{*}\right.$ ).

By certain asumptions $[9,10]$ to discuss the Degenerate Cauchy problem in Banach 
space where the space can be written in direct sum of two subspaces. The Cauchy problem in Hilbert space wich can be written as direct sum of the kernel of $M(\operatorname{Ker} M)$ and the range of adjoint $M\left(\right.$ Ran $\left.M^{*}\right)[11,12]$. The posibility of factorization and the relation of the factorized problem with the original degenerate system without asuming parabolicity [13].

\section{Nonhomogen Abstract Cauchy Problems}

Let $\mathrm{H}$ and $\mathrm{W}$ be Hilbert space [14] over $K$ complex number, and

$M: \mathrm{D}(M) \subset \mathrm{H} \rightarrow \mathrm{W} \quad ; A: \mathrm{D}(A) \subset \mathrm{H} \rightarrow \mathrm{W}$.

are linear operators. In this section we are going to investigates how to solve nobhomogen abstract degenertae Cauchy problem,

$$
\frac{d}{d t} M z(t)=A z(t)+f(t), \quad z(0)=z_{0}
$$

where $\mathrm{M}$ is not invertible. Problem (2) is called degenerate when $M$ is not invertible. The solution of (2) is defined in the following.

Definition 1: Function $z:[0, \infty) \rightarrow H$ is a strict solution of problem (2) if $z(t) \in D(A) \cap D(M)$ for all $t \geq 0, M z(t)$ is continuously differentiable, and (2) hold.

To solve the probelm we use several assumptions:

1. The operator $A, M$ are closed linear operator which are densely defined in some Hilbert space,

2. $\mathrm{PD}_{\mathrm{A}} \subset \mathrm{D}_{\mathrm{A}}$ and operator $(\mathrm{QAP}) \mid \mathrm{PD}_{\mathrm{A}}$ has bounded inverse,

3. Operator A has bounded inverse,

4. Set of $D_{A}$ is contained in domain of operator $M$,

5. Operator $M$ has a closed domain, and

6. Operator $A_{1}$ is a generator of/ Co-Semigrup di $\mathrm{H}_{0}$,

So the problem (2) can be reduced to nondegenerate abstract Cauchy $[15,16]$ problem:

$$
\begin{aligned}
\frac{d}{d t} M_{r} x(t) & =A_{0} x(t)+\left(Q^{T}-Q^{T} A P(Q A P)^{-1} Q\right) f(t) \\
& =A_{0} x(t)+Y_{A} f(t)
\end{aligned}
$$

Operator $M$ has close domain, so the problem (3) can be tranformed to problem:

$$
\frac{d}{d t} x(t)=A_{1} x(t)+\left(M_{r}\right)^{-1} Y_{A} f(t)
$$

where $A_{1}=\left(M_{r}\right)^{-1} A_{0}$.

Since the operator $A^{-1}$ is a bounded operator, so we can define $g(t)=P^{T} A^{-1} f(t)$ and then problem (4) can be written:

$\frac{d}{d t} x(t)=A_{1}(x(t)+g(t))$.

If $g(t) \in \mathscr{D}\left(A_{1}\right)$ and continuously differentiable then the solution of equation (5) is

$$
\begin{aligned}
x(t) & =e^{A_{1} t} P^{T} z_{0}+\int_{0}^{t} e^{A_{1} t(t-s)} A_{1} g(s) d s \\
& =e^{A_{1} t} P^{T} z_{0}+A_{1} \int_{0}^{t} e^{A_{1} t(t-s)} g(s) d s
\end{aligned}
$$

Lemma 2: If function $z(t)$ be solution of problem (2), then function $z(t)$ can be given by $z(t)=Z_{A} P^{T} z(t)-(Q A P)^{-1} Q f(t), \quad$ for all $t \geq 0$.

Proof : Now we will prove the lemma by reductio ad absordum. We know that $\mathrm{z}(\mathrm{t})$ is solution of (1) and let

$$
Z_{A} P^{T} z(t)-(Q A P)^{-1} Q f(t)=y(t) \text {. }
$$

So we will have:

$$
\begin{aligned}
\frac{d}{d t} M y(t) & =\frac{d}{d t} M\left[Z_{A} P^{T} z(t)-(Q A P)^{-1} Q f(t)\right] \\
& =\frac{d}{d t} M z(t)=A z(t)+f(t) .
\end{aligned}
$$

Moreover, lets $z(t) \neq y(t)$ so we have:

$$
\frac{d}{d t} M z(t) \neq \frac{d}{d t} M y(t) \neq A z(t)+f(t) \text {. }
$$

Teorem 3: Under assumptions 1, 2, 3, 4, 5 and function $f(t) \in \operatorname{Ran} M \quad$ continuously differentiable, for each $z_{0}(t) \in D_{A}$ the solution of problem (2) is given by 


$$
\begin{aligned}
& z(t)=Z_{A} x(t)-(Q A P)^{-1} Q f(t) \text { where } \\
& x(t)=e^{A_{1} t} P^{T} z_{0}+A_{1} \int_{0}^{t} e^{A_{1} t(t-s)} g(s) d s, \quad \text { and } \\
& g(s)=P^{T} A^{-1} f(s) .
\end{aligned}
$$

In order to solve nondegenerate Cauchy problem by factorization method, the operator where is located in the right handside must be generator of semigroup linear operator, so the solution of the problem is unique.

Definisi 3: Let $\mathscr{H}$ be Hilbert space and $S(t): \mathscr{H} \rightarrow \mathscr{H}$, be linear operator for $t \in R_{+}$. Semigrup $\{S(t)\}$ is set of operators $S(t): \mathcal{H} \rightarrow \mathcal{H}$, for all $t \in R_{+}$where i. $S(t+s)=S(t) S(s)$, for all $t, s \in R_{+}$.

ii. $S(0)=I$.

Moreover $\{S(t)\}$ is called strongly continuous or Co-semigrup, if $\lim _{t \rightarrow 0^{+}} S(t) x=x, \forall x \in \mathscr{H}$,

On the following lemma will be discused about differentiabelity of function $t \rightarrow S(t) x, x \in \mathcal{H}$.

Lemma 4: Let $\{S(t)\}$ be Co-semigrup on Hilbert Space $H$ and $x, y \in \mathcal{F}$. Then the following two statements are equivalent:

i. $\quad \lim _{t \rightarrow 0} \frac{1}{t}(S(t) x-x)=y$.

ii. The function $S(t)$ is differentiable for $t>0$ and $\frac{d}{d t} S(t) x=S(t) y$, for all $t>0$.

Next, if the operator $A: \mathscr{D}(A) \subset \mathscr{H} \rightarrow \mathscr{H}$, where

$$
\mathscr{D}(A)=\left\{x \in \mathfrak{F} \mid \lim _{h \rightarrow 0} \frac{1}{h}(S(h) x-x) \text { ada }\right\},
$$

then we will define infinetimal genartor of semigroup.

Definition 5: The Infinitesimal generator $A: \mathscr{D}(A) \subset \mathscr{H} \rightarrow \mathscr{H}$ of semigrup $\{S(t)\}$ is defined by:

$$
A x=\lim _{t \rightarrow+0} \frac{S(t) x-x}{t}
$$

where $x \in \mathscr{D}(A)$ if only if the limit above exists.

Every linear operator $A$ in Hilbert space does not always be generator of Co-semigrup. By the Hille-Yosida theorema we know the characteristic the linear operator is a generator of Co-semigrup[18]

Teorema 6: (Teorema Hille-Yosida) [17] Let A linear operator , $A: \mathfrak{D}(A) \subset \mathscr{H} \rightarrow \mathscr{H}, J \geq 1$ and $\omega \in R$ be constans. Then the following two statements are equivalent:

a. The operator $A$ is infinitesimal generator of Co-Semigrup.

b. The operator $A$ is closed, densely defined operator and $\left\|(A-\lambda I)^{-1}\right\| \leq \frac{J}{\operatorname{Re}(\lambda)-\omega}$,

For all $\lambda \in S$ with

$$
S=\{\lambda \in C \mid \operatorname{Re} \lambda>\omega\} \subset \rho(A) .
$$

Teorema 7: Let $\{S(t)\}$ be a Co-semigrup on Hilbert space $\mathrm{H}$ with infinitesimal generator $A$, then $x \in \mathscr{D}(A)$ implies

a. $S(t) x \in \mathscr{D}(A), \quad \forall t \geq 0$.

b. $\quad S(t) x$ is continuosly differentiable on

$$
t \geq 0 \text {, with }
$$

$$
\frac{d}{d t} S(t) x=A S(t) x=S(t) A x, \quad \forall t \geq 0 .
$$

$M$ is bounded, so $M_{r}$ is bounded and densely defined on $P^{T} \mathscr{H C}$. Since $M_{r}$ is invertible, the operator $\left(M_{r}\right)^{-1}$ is exist and bounded. Moreover we defined the operator

$$
A_{1}=\left(M_{r}\right)^{-1} A_{0}
$$

on the natural domain:

$\mathscr{D}\left(A_{1}\right)=\left\{x \in P^{T} \mathscr{D}_{A} \mid A_{0} x \in \operatorname{Ran} M\right\}=A_{0}^{-1} \operatorname{Ran} M$.

According (6), the problem nondegenerate (5) can be tranformed to canonik form:

$$
\begin{aligned}
\frac{d}{d t} x(t) & =\left(M_{r}\right)^{-1} A_{0} x(t), \quad x(0)=P^{T} z_{0} . \\
& =A_{1} x(t) .
\end{aligned}
$$

Operator $A_{1}=\left(M_{r}\right)^{-1} A_{0}$ on natural domain is closed, because it is the product of a bounded 
operator $\left(M_{r}\right)^{-1}$ and a closed operator $A_{0}$. Operator $A_{1}$ is densely defined in Hilbert space $\mathscr{H}_{0}=\overline{\left(P^{T} \mathscr{D}_{A}\right)}$.

According Theorem 7, for every $x \in \mathscr{D}(A)$ the function $u(t)=S(t) x$ is a solution of Cauchy abstract problem $\frac{d}{d t} u=A u, u(0)=x$. Moreover we must have the asumption to make the canonik problem has a unique solution.

Asumption 8: $A_{1}$ generates a strongly continuous semigroup in $\mathrm{H}_{0}$.

By assumption 8 dan Theorem 7, the solution problem (7) is $x(t)=S(t) x_{0}$ where $x_{0}=P^{T} z_{0}$.

Example 9: In this example, we have Hilbert space $\left(L^{2}(R)\right)^{2}$ and operator

$M=\left(\begin{array}{ll}1 & 0 \\ 0 & 0\end{array}\right)$ and $A=\left(\begin{array}{cc}0 & \partial / \partial x \\ \partial / \partial x & a\end{array}\right)$.

According (3), then we have

$\mathrm{D}_{A}=\left\{\left(\begin{array}{l}f \\ g\end{array}\right) \in\left(W^{1,2}(R)\right)^{2} \mid g(x)=-\frac{1}{a} f^{\prime}(x)\right\}$.

Next, we define an orthogonal projection operators:

$$
P=Q=\left(\begin{array}{ll}
0 & 0 \\
0 & 1
\end{array}\right) \text { dan } P^{T}=Q^{T}=\left(\begin{array}{ll}
1 & 0 \\
0 & 0
\end{array}\right)
$$

By the projection operators, we have operator:

$$
\begin{aligned}
& M_{r}=M P^{T}=\left(\begin{array}{ll}
1 & 0 \\
0 & 0
\end{array}\right) \text { and } \\
& A_{0}=Y_{A} A=A P^{T}=\left(\begin{array}{cc}
-\frac{\partial^{2}}{a \partial x^{2}} & 0 \\
0 & 0
\end{array}\right) .
\end{aligned}
$$

In this case, operator $A_{0}=-\frac{1}{a} \partial^{2} / \partial x^{2}$ on $W^{2,2}(R)$ and $M_{r}=1$ on $L^{2}(R)$. So the solution of this problem is
$z(t)=Z_{A} x(t)$, where

$$
x(t)=e^{A_{1} t} P^{T} z(0), Z_{A}=\left(\begin{array}{cc}
1 & 0 \\
-\frac{\partial}{a \partial x} & 0
\end{array}\right) .
$$

\section{Conclusion}

After we discuss this topic, we can result how to solve degenerate abstract Cauchy problem by semigroup theory of linear operator. There are three stages to solve this problem. In the first stage, under certain assumptions we reduce the nonhomogen abstract degenertae Cauchy problem (2):

$$
\frac{d}{d t} M z(t)=A z(t)+f(t), \quad z(0)=z_{0}
$$

to nondegenerate Cauchy problem (3):

$\frac{d}{d t} M_{r} x(t)=A_{0} x(t)+Y_{A} f(t), x(t)=P^{T} z_{0}$.

Operator $M$ which is not invertible, can be reduced to invertible operator $M_{r}$.

The second stage the problem (3) can be tranformed to canonik form:

$$
\frac{d}{d t} x(t)=A_{1} x(t)+\left(M_{r}\right)^{-1} Y_{A} f(t)
$$

where $A_{1}=\left(M_{r}\right)^{-1} A_{0}$.

In order to use semigroup theory of linear operator, we assume that $\mathrm{A}_{1}$ is an infinitesimal generator of strongly continuous semigroup, so the solution is

$$
x(t)=e^{A_{1} t} P^{T} z_{0}+A_{1} \int_{0}^{t} e^{A_{1} t(t-s)} g(s) d s
$$

The third, finally by $Z_{A}$ we can find a solution of the original problem is

$$
z(t)=Z_{A} x(t)-(Q A P)^{-1} Q f(t)
$$

and $g(s)=P^{T} A^{-1} f(s)$. 


\section{Acknowledgment}

The author wish to thank Mathematics Department, Faculty of Mathematics and Natural Science, Universitas Diponegoro.

\section{References}

[1] L. Dai, Singular Control Systems, Lecture Notes in Control and Inform, Sci., 118. 1989.

[2] R. W. Carroll, R. E Showalter, Singular and Degenerate Cauchy Problems. Math. Sci. Engrg., 127, 1976.

[3] A. Favini, Controllability Condition of Linear degenerate Evolution Systems. Appl. Math. Optim. 1980.

[4] A. Favini, A Degenerate and Singular Evolution Equations in Banach Space. Math. Ann., 273, 1985.

[5] A. Favini, A.. Abstract Potential Operator and Spectral Method for a Class of Degenerate Evolution Problems. J. Differential Equations, 39, 1981.

[6] A. Favini, P. Plazzi, On Some Abstract Degenerate Problems of Parabolic Type1 the Linear Case. Nonlinear Analysis, 12, 1988.

[7] A. Favini, P. Plazzi, On Some Abstract Degenerate Problems of Parabolic Type2 theNonlinear Case. Nonlinear Analysis, 13, 1989.

[8] A. Favini, P. Plazzi, P. On Some Abstract Degenerate Problems of Parabolic Type-3 Applications to Linear and Nonlinear Problems. Osaka J. Math, 27, . 1990.
[9] A. Favini,. Laplace Tranform Method for a Class of Degenerate Evolution Problems. Rend. Mat. Appl. 2, pp.12, 1979.

[10] A. Favini, A. Yagi, Space and Time Regularity for Degenerate Evolution Equations. J. Math. Soc. Japan, 44, 1992.

[11] B.Thaller, S. Thaller, Approximation of Degenerate Cauchy Problems, SFB F0003 "Optimierung und Kontrolle" 76, 1996.

[12] B. Thaller, The Dirac Eqution, Text and Monographs in Physics, Heidelberg-New York: Springer Verlag, 1992.

[13] B.Thaller, S. Thaller,. Factorization of Degenerate Cauchy Problems : The Linear Case. Operator Theory Journal, pp. 121-146, 1996.

[14] J. Weidman, Linear Operators in Hilbert Spaces, 1980.

[15] M. Hernandez Existence Result For Second-Order Abstract Cauchy Problem With NonLocal Conditions. Electronic Journal of Differential Equations, 2005.

[16] E. Zeidler, Nonlinear Functional Analysis and Its Applications II/A. 1990.

[17] F. Kappel, ,W. Schappacher, Strongly Continuous Semigroups, An Introduction. 2000.

[18] A. Pazy, Semigroups of Linear Operators and Applications to Partial Differential Equations, 1983. 